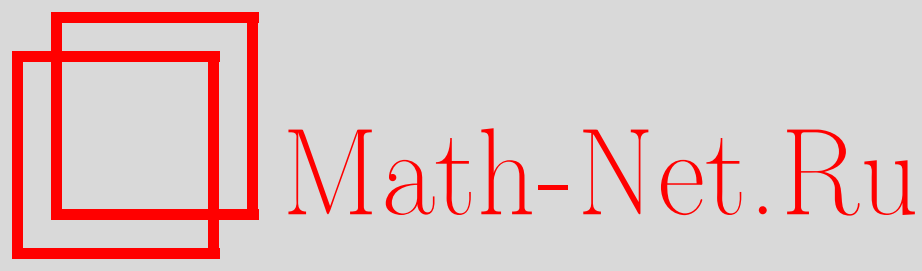

А. Х. Хачатрян, Х. А. Хачатрян, Качественные различия решений для одной модели уравнения Больцмана в линейном и нелинейном случаях, ТМФ, 2012, том 172 , номер 3, 497-504

DOI: https://doi.org/10.4213/tmf6965

Использование Общероссийского математического портала Math-Net.Ru подразумевает, что вы прочитали и согласны с пользовательским соглашением http://www.mathnet.ru/rus/agreement

Параметры загрузки:

IP : 18.234 .197 .8

26 апреля 2023 г., $11: 07: 56$

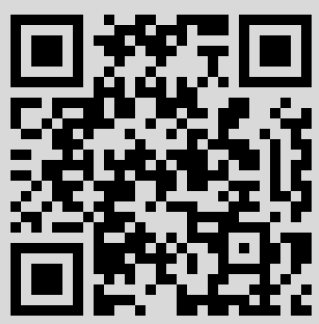


Том 172, № 3

сентябрь, 2012

(C) 2012 г. $\quad$ A.X. Хачатрян ${ }^{*}$, Х. А. Хачатрян

\title{
КАЧЕСТВЕННЫЕ РАЗЛИЧИЯ РЕШЕНИЙ ДЛЯ ОДНОЙ МОДЕЛИ УРАВНЕНИЯ БОЛЬЦМАНА В ЛИНЕЙНОМ И НЕЛИНЕЙНОМ СЛУЧАЯХ
}

\begin{abstract}
Рассматривается уравнение Больцмана в рамках одной нелинейной модели для задач течения газа в полупространстве (задача Крамерса). Доказывается существование положительного и ограниченного решения, найден предел этого решения в бесконечности. Показано, что учет нелинейной зависимости интеграла столкновения от функции распределения приводит к асимптотически новому решению исходного уравнения. Для иллюстрации результата приведены примеры функций, описывающих нелинейность интеграла столкновения.
\end{abstract}

Ключевые слова: уравнение Больцмана, нелинейность, среднемассовая скорость, ограниченное решение, монотонность.

\section{1. ВВЕДЕНИЕ}

Как известно, в основе физической кинетики лежит уравнение Больцмана (УБ), которое первоначально было создано в основном для описания кинетики разреженного газа, а в дальнейшем стало предметом исследования в различных областях физики [1]-[6]. В кинетической теории разреженного газа важное место занимает круг задач, связанных с изучением течения газа в полупространстве, ограниченном твердой стенкой (задача Крамерса), а также течения газа между двумя параллельными пластинками (задача Куэтта) [1], [2]. При этом молекулы газа взаимодействуют между собой по определенным внутриатомным законам, а некоторая часть из них взаимодействует со стенкой, что и обусловливает нелинейную зависимость интеграла столкновения от функции распределения частиц. Поэтому для УБ разработаны различные модели, среди которых особое место занимает модель Бхатнагара-Гросса-Крука (БГК) [7]. В этой модели интеграл столкновения $\operatorname{st} f$ заменяется $f_{0}^{\text {loc }}-f$, где $f$ - искомая функция распределения $(\Phi \mathrm{P}), f_{0}^{\text {loc }}$ локально-равновесная функция Максвелла. Применение этой модели сопровождается приближенной линеаризацией полученного нелинейного уравнения. При этом

*Армянский государственный аграрный университет, Ереван, Армения.

E-mail: Aghavard@hotbox.ru

${ }^{\dagger}$ Институт математики НАН Республики Армения, Ереван, Армения.

E-mail: Khach82@rambler.ru 
в предположении, что среднемассовая скорость газа намного меньше молекулярной скорости, локально-равновесная функция Максвелла заменяется суммой первых двух членов разложения в степенной ряд. В случае задач Крамерса такая линеаризация приводит к тому, что среднемассовая скорость $u(x)$ неограниченно растет как $O(x)$ при $x \rightarrow+\infty$ (где $x$ - расстояние от стенки). Это противоречит вышеприведенному предположению о малости среднемассовой скорости. Оказывается, что линеаризация может существенно исказить задачу как в количественном, так и в качественном отношении, т. е. привести к асимптотически иному решению, которое отличается от решения исходного нелинейного интегродифференциального уравнения.

В настоящей работе на примере одной нелинейной модели для задачи Крамерса доказывается существование положительного и ограниченного решения, найден предел этого решения в бесконечности. В рамках предложенной нами модели приводятся примеры функций, описывающих нелинейность интеграла столкновения.

\section{2. ПОСТАНОВКА ЗАДАЧИ}

Введем в $\mathbb{R}^{3}$ декартову систему координат $(x ; y ; z)$, и пусть газ заполняет полупространство $x>0$, ограниченное твердой стенкой-плоскостью $x=0$.

Будем предполагать, что газ стационарно течет по положительному направлению оси $O Y$. Пусть $f(x, \vec{s})$ - искомая $Ф$ Р частиц по скоростям $\vec{s}\left(s_{1}, s_{2}, s_{3}\right)$. В силу предполагаемой плоской симметрии задачи функция $f(x, \vec{s})$ не зависит от $y$ и $z$. УБ в рамках предложенной нами нелинейной модели для безразмерной задачи Крамерса записывается в следующей форме:

$$
s_{1} \frac{\partial f(x, \vec{s})}{\partial x}+f(x, \vec{s})=f_{0}(\vec{s})\left(1+2 s_{2} R(u(x))\right) .
$$

Здесь $f_{0}(\vec{s})=\left(1 / \pi^{3 / 2}\right) e^{-s^{2}}-$ безразмерная ФР Максвелла, $u(x)-$ среднемассовая скорость газа, которая связана с $\Phi$ P $f(x, \vec{s})$ :

$$
u(x)=\int_{-\infty}^{+\infty} \int_{-\infty}^{+\infty} \int_{-\infty}^{+\infty} s_{2} f(x, \vec{s}) d^{3} s
$$

функция $R(\tau)$ описывает нелинейную зависимость от $\Phi \mathrm{P}$, и условия, налагаемые на нее, приводятся ниже (см. теорему 1). Заметим, что в случае $R(\tau) \equiv \tau$ мы приходим к задаче Крамерса для линеаризованной БГК-модели УБ.

Представим искомую ФР в виде

$$
f(x, \vec{s})=f_{0}(\vec{s})(1+h(x, \vec{s})),
$$

где $h(x, \vec{s})$ - искомая $\Phi$, являющаяся поправкой к безразмерной ФР Максвелла, вызванной присутствием стенки. На функцию $f(x, \vec{s})$ налагается также условие непротекания:

$$
\int_{-\infty}^{+\infty} \int_{-\infty}^{+\infty} \int_{-\infty}^{+\infty} s_{1} f(x, \vec{s}) d^{3} s=0 .
$$


Тогда уравнение (1) преобразуется к виду

$$
s_{1} \frac{\partial h(x, \vec{s})}{\partial x}+h(x, \vec{s})=2 s_{2} R(u(x))
$$

Обозначим через $h^{-}(x, \vec{s})$ и $h^{+}(x, \vec{s})$ функции, описывающие распределение по скоростям для молекул, двигающихся соответственно в сторону стенки и обратно:

$$
h^{-}(x, \vec{s})=\left\{\begin{array}{ll}
0, & \text { если } s_{1}<0, \\
h\left(x,-s_{1}, s_{2}, s_{3}\right), & \text { если } s_{1} \geqslant 0,
\end{array} \quad h^{+}(x, \vec{s})= \begin{cases}h(x, \vec{s}), & \text { если } s_{1} \geqslant 0 \\
0, & \text { если } s_{1}<0 .\end{cases}\right.
$$

Умножая обе части уравнения $(3)$ на $(1 / \pi) s_{2} e^{-s_{2}^{2}} e^{-s_{3}^{2}}$ и интегрируя по $s_{2}$ и $s_{3}$ от $-\infty$ до $+\infty$, получим

$$
s \frac{\partial \varphi(x, s)}{\partial x}+\varphi(x, s)=R(u(x)), \quad x>0
$$

Здесь $s \equiv s_{1}$,

$$
\begin{gathered}
u(x)=\frac{1}{\sqrt{\pi}} \int_{0}^{\infty} e^{-s^{2}}\left[\varphi^{+}(x, s)+\varphi^{-}(x, s)\right] d s, \\
\varphi(x, s)=\frac{1}{\pi} \int_{-\infty}^{+\infty} \int_{-\infty}^{+\infty} s_{2} e^{-s_{2}^{2}} e^{-s_{3}^{2}} h\left(x, s, s_{2}, s_{3}\right) d s_{2} d s_{3}, \\
\varphi^{+}(x, s)=\left\{\begin{array}{ll}
\varphi(x, s), & \text { если } s \geqslant 0, \\
0, & \text { если } s<0,
\end{array} \quad \varphi^{-}(x, s)= \begin{cases}0, & \text { если } s<0, \\
\varphi(x,-s), & \text { если } s \geqslant 0 .\end{cases} \right.
\end{gathered}
$$

K уравнению (5) присоединим граничные условия на стенке:

$$
\begin{gathered}
\varphi^{+}(0, s)=\int_{0}^{\infty} Q\left(s, s^{\prime}\right) \varphi^{-}\left(0, s^{\prime}\right) d s^{\prime}, \quad s>0 \\
\varphi^{-}(x, s)=o\left(e^{x / s}\right), \quad x \rightarrow+\infty
\end{gathered}
$$

Здесь функция $Q\left(s, s^{\prime}\right)$ описывает закон отражения от стенки.

Из уравнения (5) с учетом условия (9) имеем

$$
\begin{aligned}
& \varphi^{+}(x, s)=c e^{-x / s}+\int_{0}^{x} e^{-(x-t) / s} R(u(t)) \frac{d t}{s}, \\
& \varphi^{-}(x, s)=\int_{x}^{\infty} e^{-(t-x) / s} R(u(t)) \frac{d t}{s} .
\end{aligned}
$$


Из уравнения (6) с учетом формул (8), (10) и (11) получим

$$
u(x)=\int_{0}^{\infty} K(x-t) R(u(t)) d t+\int_{0}^{\infty} K_{1}(x, t) R(u(t)) d t,
$$

где

$$
K_{1}(x, t)=\frac{1}{\sqrt{\pi}} \int_{0}^{\infty} e^{-x / s} e^{-s^{2}} d s \int_{0}^{\infty} \frac{Q\left(s, s^{\prime}\right)}{s^{\prime}} e^{-t / s^{\prime}} d s^{\prime}
$$

a

$$
K(x)=\frac{1}{\sqrt{\pi}} \int_{0}^{\infty} e^{-|x| / s} e^{-s^{2}} \frac{d s}{s}
$$

- известное ядро Веландера [1], [2].

Нетрудно проверить, что выполняется условие консервативности:

$$
K(x) \geqslant 0, \quad x \in \mathbb{R}^{+}, \quad \int_{-\infty}^{+\infty} K(x) d x=1 .
$$

Заметим, что в частном случае $Q\left(s, s^{\prime}\right)=\varepsilon \delta\left(s-s^{\prime}\right)(\delta$ - обобщенная функция Дирака) $K_{1}(x, t)$ становится зависящим от суммы аргументов и $K_{1}(x) \equiv \varepsilon K(x)$. При этом предполагается, что часть частиц $\varepsilon, 0 \leqslant \varepsilon<1$, отражается от стенки зеркально, а остальная часть $1-\varepsilon$ поглощается стенкой и затем изотропно переизлучается. Величина $\varepsilon$ называется коэффициентом аккомодации.

\section{3. ОСНОВНОЙ РЕЗУЛЬТАТ}

Рассмотрим нелинейное интегральное уравнение (12) относительно искомой измеримой и вещественной функции $u(x)$, где $K(x)$ и $K_{1}(x, t)$ задаются посредством формул $(13),(14)$. Функция $Q\left(s, s^{\prime}\right)$, определенная на множестве $\mathbb{R}^{+} \times \mathbb{R}^{+},-$измеримая функция, причем

$$
Q\left(s, s^{\prime}\right) \geqslant 0, \quad\left(s, s^{\prime}\right) \in \mathbb{R}^{+} \times \mathbb{R}^{+}, \quad \int_{0}^{\infty} Q\left(s, s^{\prime}\right) d s^{\prime} \leqslant 1 .
$$

Поскольку взаимодействие частиц со стенкой не сопровождается потерей или "рождением" новых частиц, то граничное условие должно быть консервативным. Этот факт отражается в условии (15).

Пусть $R$ - описывающая нелинейность уравнения (12), измеримая и вещественная функция, определенная на $(-\infty,+\infty)$. Пусть числа $\xi$ и $\eta, 2 \xi<\eta,-$ первые положительные корни уравнений $R(x)=2 x$ и $R(x)=x$ соответственно. Далее предполагается, что $R \in C[\xi, \eta], R \uparrow$ на отрезке $[\xi, \eta], R(0)=0$.

Обозначим через $M(0,+\infty)$ пространство измеримых и существенно ограниченных функций на $(0,+\infty)$. Тогда справедлива следующая теорема.

ТЕОРема 1. Пусть выполнены вышеприведенные условия относительно функций $K, Q$ и $R$. Тогда уравнение (12) имеет положительное решение из пространства $M(0,+\infty)$. Более того,

$$
\lim _{x \rightarrow+\infty} u(x)=\eta .
$$


ДокАЗАТЕЛЬСтво. Рассмотрим следующие итерации:

$$
\begin{aligned}
& u_{n+1}(x)=\int_{0}^{\infty} K(x-t) R\left(u_{n}(t)\right) d t+ \\
& +\frac{1}{\sqrt{\pi}} \int_{0}^{\infty} e^{-x / s} e^{-s^{2}}\left[\int_{0}^{\infty} Q\left(s, s^{\prime}\right) \int_{0}^{\infty} e^{-t / s^{\prime}} R\left(u_{n}(t)\right) \frac{d t}{s^{\prime}} d s^{\prime}\right] d s \\
& u_{0}(x) \equiv \eta, \quad n=0,1,2, \ldots, \quad x>0 .
\end{aligned}
$$

Ниже мы докажем, что

$$
\begin{gathered}
u_{n}(x) \downarrow \text { по } n, \quad x>0, \\
u_{n}(x) \geqslant \xi, \quad n=0,1,2, \ldots, \quad x>0 .
\end{gathered}
$$

Сначала докажем утверждение (19). Используя соотношения (14) и (15), из формулы (17) получим

$$
\begin{aligned}
u_{1}(x)= & \int_{0}^{\infty} K(x-t) R(\eta) d t+ \\
& +\frac{1}{\sqrt{\pi}} \int_{0}^{\infty} e^{-x / s} e^{-s^{2}}\left(\int_{0}^{\infty} \frac{1}{s^{\prime}} Q\left(s, s^{\prime}\right) \int_{0}^{\infty} e^{-t / s^{\prime}} R(\eta) d t d s^{\prime}\right) d s= \\
= & \frac{\eta}{\sqrt{\pi}} \int_{0}^{\infty} \int_{0}^{\infty} e^{-|x-t| / s} e^{-s^{2}} \frac{d t d s}{s}+\frac{\eta}{\sqrt{\pi}} \int_{0}^{\infty} e^{-s^{2}} e^{-x / s}\left(\int_{0}^{\infty} Q\left(s, s^{\prime}\right) d s^{\prime}\right) d s \leqslant \\
\leqslant & \frac{\eta}{\sqrt{\pi}} \int_{0}^{\infty} \int_{0}^{\infty} e^{-|x-t| / s} e^{-s^{2}} \frac{d t d s}{s}+\frac{\eta}{\sqrt{\pi}} \int_{0}^{\infty} e^{-s^{2}} e^{-x / s} d s= \\
= & \eta \int_{-\infty}^{+\infty} K(\tau) d \tau=\eta=u_{0}(x) .
\end{aligned}
$$

Предполагая, что $u_{n}(x) \geqslant u_{n+1}(x)$, из монотонности функции $R$ получим $u_{n+1}(x) \geqslant$ $u_{n+2}(x)$.

Теперь докажем неравенства (20). При $n=0$ последнее сразу следует из условия теоремы. Предположим, что $u_{n}(x) \geqslant \xi$ при некотором $n \in \mathbb{N}$. Тогда в силу свойств функций $R$ и $K$ из формулы (17) получаем

$$
u_{n+1}(x) \geqslant \int_{0}^{\infty} K(x-t) R(\xi) d t=2 \xi \int_{-\infty}^{x} K(z) d z \geqslant \xi
$$

(ибо $\int_{-\infty}^{0} K(z) d z=1 / 2$ ). Следовательно, существует предел последовательности $\left\{u_{n}(x)\right\}_{n=0}^{\infty}$

$$
\lim _{n \rightarrow \infty} u_{n}(x)=u(x)
$$

причем этот предел по теореме Леви удовлетворяет уравнению (12). Кроме того,

$$
\xi \leqslant u(x) \leqslant \eta .
$$

Докажем, что $\lim _{x \rightarrow \infty} u(x)=\eta$. С этой целью рассмотрим усеченное нелинейное уравнение

$$
f(x)=\int_{0}^{\infty} K(x-t) R(f(t)) d t, \quad x>0,
$$


относительно функции $f(x)$ и следующие итерации для него:

$$
f_{n+1}(x)=\int_{0}^{\infty} K(x-t) R\left(f_{n}(t)\right) d t, \quad f_{0}(x) \equiv \eta, \quad n=0,1,2, \ldots
$$

Индукцией по $n$ нетрудно убедиться, что
1) $f_{n}(x) \downarrow$ по $n$,
2) $f_{n}(x) \geqslant \xi$,
3) $f_{n}(x) \leqslant u_{n}(x)$
$n=1,2,3, \ldots$.

Записывая итерации (25) в виде

$$
f_{n+1}(x)=\int_{-\infty}^{x} K(\tau) R\left(f_{n}(x-\tau)\right) d \tau, \quad f_{0}(x) \equiv \eta, \quad n=0,1,2, \ldots,
$$

индукцией по $n$ нетрудно показать, что

$$
f_{n}(x) \uparrow \text { по } x \text { на }(0,+\infty), \quad n=0,1,2, \ldots .
$$

Следовательно, существует $\lim _{n \rightarrow \infty} f_{n}(x)=f(x)$, причем из формулы (27) следует, что

$$
f(x) \uparrow \text { по } x \text { на }(0,+\infty),
$$

а из (26) получим

$$
\xi \leqslant f(x) \leqslant u(x) \leqslant \eta
$$

Из выражения (28) с учетом (29) следует существование предела

$$
\lim _{x \rightarrow \infty} f(x)=\eta_{0}>0
$$

В уравнении (24), учитывая непрерывность функции $R$ и в силу известных свойств операций свертки, будем иметь

$$
\eta_{0}=R\left(\eta_{0}\right)
$$

Так как $\eta$ - первый положительный корень уравнения $R(x)=x$, то $\eta_{0}=\eta$. Таким образом,

$$
\lim _{x \rightarrow \infty} f(x)=\eta
$$

Учитывая неравенство (29), в силу равенства (31) приходим к соотношению

$$
\lim _{x \rightarrow \infty} u(x)=\eta
$$

Теорема доказана.

ЗАмечАниЕ 1. Ниже на одном простом примере покажем, что решение соответствующей линейной задачи (в отличие от нелинейной) имеет линейный рост в бесконечности.

Рассмотрим основное уравнение (12) в линейном случае, т. е. когда $R(\tau)=\tau$. Пусть $Q\left(s, s^{\prime}\right)=\varepsilon \delta\left(s-s^{\prime}\right)$. 
Тогда из уравнения (12) будем иметь

$$
u(x)=\int_{0}^{\infty} K(x-t) u(t) d t+\varepsilon \int_{0}^{\infty} K(x+t) u(t) d t .
$$

Изучению и точному решению консервативных однородных линейных интегральных уравнений типа (33) посвящена работа [8]. Из результатов работ [8] и [9] следует, что уравнение (33) обладает положительным решением с асимптотикой $O(x)$ при $x \rightarrow \infty$.

Таким образом, среднемассовая скорость $u(x)$ в линейном приближении имеет асимптотику $O(x)$ при $x \rightarrow \infty$, в то время как учет нелинейной зависимости интеграла столкновения приводит к ограниченному решению.

ЗАмЕчАниЕ 2. Следует отметить, что вышеизложенный подход легко можно распространить на задачи переноса излучения монохроматического рассеяния в плоском слое при наличии отражающей поверхности.

ЗАмЕчАНИЕ 3. Отметим, что результаты также остаются в силе, если учесть нелинейный закон отражения от стенки, т. е. вместо условия (8) исследовать следующее граничное условие:

$$
\varphi^{+}(0, s)=R_{1}\left(\int_{0}^{\infty} Q\left(s, s^{\prime}\right) \varphi^{-}\left(0, s^{\prime}\right) d s^{\prime}\right), \quad s>0
$$

где на функцию $R_{1}(\tau)$ налагаются условия

$$
R_{1} \in C[0, \eta], \quad R_{1} \uparrow \quad \text { на }[0, \eta], \quad 0 \leqslant R_{1}(\tau) \leqslant \tau, \quad \tau \in[0, \eta] .
$$

В качестве функции $R(\tau)$ можно привести несколько примеров:

$$
\begin{gathered}
\text { 1) } \left.\quad R(\tau)=\tau^{\alpha}, \quad \alpha \in(0 ; 1) ; \quad 2\right) \quad R(\tau)=\sqrt{\tau e^{\tau-1}} \\
\text { 3) } \quad R(\tau)=\sqrt{\tau(\tau+\sin \tau+1)}
\end{gathered}
$$

Например, уравнение (12) со степенной нелинейностью (первый пример) принимает следующий вид:

$$
\begin{gathered}
u(x)=\int_{0}^{\infty} K(x-t) u^{\alpha}(t) d t+\int_{0}^{\infty} K_{1}(x, t) u^{\alpha}(t) d t \\
\alpha \in(0,1), \quad x>0 .
\end{gathered}
$$

В этом случае корни уравнений $R(x)=2 x$ и $R(x)=x$ будут $\xi=(1 / 2)^{1 /(1-\alpha)}$ и $\eta=1$ соответственно. Следовательно, из доказанной теоремы 1 следует, что при условии (15) уравнение (36) с ядрами (13), (14) имеет положительное решение со свойствами

$$
\left(\frac{1}{2}\right)^{1 /(1-\alpha)} \leqslant u(x) \leqslant 1, \quad \lim _{x \rightarrow \infty} u(x)=1 .
$$

Благодарности. Авторы выражают благодарность рецензенту за полезные замечания. 


\section{Список литературы}

[1] М.Н. Коган, Динамика разреженного газа. Кинетическая теория, Наука, М., 1967.

[2] C. Cercignani, The Boltzmann Equation and its Applications, Applied Mathematical Sciences, 67, Springer, New York, 1988.

[3] А.В. Латышев, А.А. Юшканов, Ж. вычисл. матем. и матем. физ., 41:8 (2001), $1229-1241$.

[4] Н. Б. Енгибарян, А. Х. Хачатрян, ТМФ, 125:2 (2000), 339-342.

[5] C. E. Siewert, J. R. Thomas Jr., Phys. Fluids, 16:9 (1973), 1557-1559.

[6] L. B. Barichello, C. E. Siewert, European J. Appl. Math., 11:4 (2000), 353-364.

[7] P. L. Bhatnagar, E. P. Gross, M. Krook, Phys. Rev., 94:3 (1954), 511-525.

[8] Н.Б. Енгибарян, А.Х. Хачатрян, Ж. вычисл. матем. и матем. физ., 38:3 (1998), $466-482$.

[9] Л. Г. Арабаджян, Н. Б. Енгибарян, "Уравнения в свертках и нелинейные функциональные уравнения", Итоги науки и техн. Сер. Мат. анал., 22, ВИНИТИ, М., 1984, 175-244.

Поступила в редакцию 27.01.2012 